\title{
Muscle Analysis by Measurement of Maximal Isometric Grip Force: New Reference Data and Clinical Applications in Pediatrics
}

\author{
FRANK RAUCH, CHRISTINA M. NEU, GERNOT WASSMER, BODO BECK, \\ GABRIELE RIEGER-WETTENGL, ERNST RIETSCHEL, FRIEDRICH MANZ, AND ECKHARD SCHOENAU \\ Children's Hospital [F.R., C.M.N., B.B., G.R.-W., E.R., E.S.] and Institute of Medical Statistics [G.W.], \\ University of Cologne, Cologne, Germany, and Research Institute of Child Nutrition, Dortmund, Germany \\ [C.M.N., F.M.]
}

\begin{abstract}
ABST
Skeletal muscle development is one of the key features of
childhood and adolescence. Determining maximal isometric grip
force (MIGF) using a hand-held Jamar dynamometer is a simple
method to quantify one aspect of muscle function. Presently
available reference data present MIGF as a function of chrono-
logical age. However, muscle force is largely determined by
body size, and many children undergoing muscle performance
tests in the clinical setting suffer from growth retardation sec-
ondary to a chronic disorder. Reference data were established
from simple regressions between age or log height and log MIGF
in a population of 315 healthy children and adolescents aged 6 to
19 y (157 girls). These data were used to calculate age- or
height-dependent SD scores (SDS) for MIGF in three pediatric
patient groups. In renal graft recipients ( $n=14)$, the age-
dependent MIGF SDS was markedly decreased $(-2.5 \pm 1.9$;
mean \pm SD). However, these patients had short stature (height
\end{abstract}
Skeletal muscle development is one of the key features of childhood and adolescence. Muscle action determines the functional requirements that are imposed on other organ systems, such as lungs, heart, and the skeleton (1). Despite its fundamental importance for development, the muscle system appears to receive relatively little consideration in pediatric science and practice. In the pediatric literature, few studies consider the influence of diseases or therapies on the muscle system. In clinical practice, analysis of muscle force is typically limited to the subjective impression of the pediatrician. A more quantitative measure of muscle function can be gained by determining MIGF using a hand-held dynamometer. This is a simple, cheap, and well-established method to quantify one aspect of muscle function. Probably the most widely used dynamometer is the Jamar device, which has been in use for decades (2).

Received April 3, 2001; accepted August 23, 2001.

Correspondence and reprint requests: Frank Rauch, M.D., Shriners Hospital for Children, 1529 Cedar Avenue, Montreal, Qc H3G 1A6, Canada; e-mail: frauch@shriners.mcgill.ca
SDS, $-2.5 \pm 1.2$ ), and the height-dependent MIGF SDS was close to normal $(-0.4 \pm 1.5)$. Similarly, in cystic fibrosis patients $(n=13)$ age-dependent MIGF SDS was $-1.6 \pm 1.6$, but height-dependent MIGF SDS was $-0.5 \pm 1.1$. Children with epilepsy who were taking anticonvulsant therapy $(n=34)$ had normal stature, and consequently age- and height-dependent MIGF SDS were similar ( $0.4 \pm 1.0$ and $0.4 \pm 0.8$, respectively). In conclusion, MIGF determination provides information on an important aspect of physical development. Height should be taken into account to avoid misinterpretation. (Pediatr Res 51: 505-510, 2002)

\section{Abbreviations}

MIGF, maximal isometric grip force SDS, SD score

Several authors have published pediatric reference data for this equipment (3-6). The practical utility of these reports is limited by the fact that they present MIGF data as a function of age. From a functional perspective the muscle system needs to be adapted to body size rather than to age. Indeed, muscle force is largely determined by body size (7-9). However, many children and adolescents undergoing muscle performance tests in the clinical setting suffer from growth retardation secondary to a chronic disorder. Using age-dependent reference data in such a situation may lead to the conclusion that a muscle disorder is present, whereas in fact low MIGF could simply be the result of a smaller body frame. If the effect of a disease on the muscle system is to be evaluated, it is therefore important to examine whether the disease has an impact on MIGF in addition to its effect on height.

In this study we determined MIGF in a large and wellcharacterized group of healthy children and young adults. The aim of this study was to establish reference material for MIGF that could be used in the clinical setting. This was exemplified in three pediatric patient groups: children and adolescents who 
had undergone kidney transplantation, patients with cystic fibrosis, and patients with epilepsy who were taking anticonvulsant therapy.

\section{METHODS}

Healthy subjects. The healthy study population comprised 315 healthy children and adolescents aged 6 to 19 y (157 girls, 148 boys). The children were participants in the DONALD (Dortmund Nutritional and Anthropometric Longitudinally Designed) Study, an ongoing observational study investigating the interrelations of nutrition, growth, and metabolism in healthy children. This study is performed at the Research Institute for Child Nutrition in Dortmund, Germany. The cohort was initially recruited for an anthropometric study in a representative sample of school children of Dortmund and later through personal recommendation of parents whose children were already participating. Overall, the study population mostly comprised middle-class families, and all participants were of white origin. On an annual basis, all participants undergo a full medical history and examination starting in infancy. MIGF testing was performed once in each participant on the occasion of a yearly follow-up visit.

The stage of pubertal development was determined in all study participants by physical examination using the grading system defined by Tanner for pubic hair (10). Height was determined to the next succeeding $1 \mathrm{~mm}$ using a Harpenden stadiometer. Weight was measured to the nearest $0.1 \mathrm{~kg}$ using digital electronic scales with the children clothed in underwear. Age at testing was calculated to two decimals. Informed consent was obtained from the children's parents or from the subjects aged $18 \mathrm{y}$ or older. In addition, written assent was also obtained from subjects between 14 and $17 \mathrm{y}$ of age. The study protocol was approved by the Ethics Committee of the University of Cologne.

Patient groups. The patient population comprised ambulatory outpatients of the Cologne University Children's Hospital, who fell into one of the three diagnostic groups described below. Inclusion criteria were age between 7 and 19 y and body height between 120 and $170 \mathrm{~cm}$ for girls and between 120 and $180 \mathrm{~cm}$ for boys (see results in healthy populations for the justification of these cutoff values). MIGF was tested in successive patients fulfilling these criteria.

The kidney transplantation group $(n=14$; three girls, 11 boys) included patients who had received a renal graft from 3 mo to $9 \mathrm{y}$ before the present study (median, $3 \mathrm{y}$ ). Current creatinine clearance ranged from $11 \mathrm{~mL} \cdot \mathrm{min}^{-1} \cdot 1.73 \mathrm{~m}^{-2}$ ) to $111 \mathrm{~mL} \cdot \mathrm{min}^{-1} \cdot 1.73 \mathrm{~m}^{-2}$ ) with a median of $74 \mathrm{~mL} \cdot \mathrm{min}^{-1} \cdot 1.73$ $\left.\mathrm{m}^{-2}\right)$. Patients with cystic fibrosis $(n=13$; six girls, seven boys) were receiving standard clinical care including intensive physical therapy. Patients with epilepsy who were taking anticonvulsants ( $n=34$; 16 girls, 18 boys) had received monotherapy with either valproic acid or carbamazepine with serum levels in the therapeutic range for at least $1 \mathrm{y}$ (median, $3 \mathrm{y}$; range, $1-9 \mathrm{y}$ ), and had a normal neurologic status.

MIGF testing. Maximal isometric MIGF of the nondominant hand was determined with a standard adjustable-handle Jamar dynamometer (Preston, Jackson, MI, U.S.A.). The han- dle was adjusted so that the line of the subject's proximal interphalangeal joints rested exactly on top of the adjustable handle. Consequently, setting 1 of the Jamar dynamometer was used for the younger children, setting 2 for most of the older children and adolescents. Setting 3 was used in some of the adolescents, whereas settings 4 and 5 were never used.

The subjects were seated with their shoulder adducted and neutrally rotated. The dynamometer was held freely, without support. The elbow was flexed at $90^{\circ}$, and care was taken that it did not touch the trunk. The forearm was in a neutral position, and the wrist was held at between $0^{\circ}$ and $30^{\circ}$ dorsiflexion and between $0^{\circ}$ and $15^{\circ}$ ulnar deviation. The subjects were told to put maximal force on the dynamometer. The maximal value of two trials was noted. The scale of the dynamometer indicates the result in kilograms, which is incorrect, as this is the unit of mass, not force. MIGF (unit in Newtons) was calculated by multiplying the dynamometer reading by a factor of 9.81 . We use the term "grip force" instead of the more widely used "grip strength," because "force" is a term that is clearly defined by physics, whereas "strength" is used inconsistently in the medical literature, denoting a variety of different measurements, including force, torque, and power.

Statistical analysis. All results in patient groups were compared with data obtained in our reference population. Height and weight of patients were converted into age- and sexspecific SDS using the following formula:

$$
\begin{aligned}
& \text { SDS }=[\text { (test result for a patient }) \\
& \quad-(\text { age- and sex-specific mean in reference population })] /
\end{aligned}
$$

(age-specific SD in reference population).

A detailed description of how MIGF reference data were computed is given in the "Results" section. The MIGF result of each patient was transformed to an age- or height-dependent SDS. Based on the patient's age or height, an expected value of $\log$ MIGF was calculated from the regression equations obtained in healthy children. This expected value was subtracted from the logarithmically transformed MIGF reading of the patient, and the result was divided by the residual SD of the regression equation for the healthy group.

The mean SDS in a healthy population should be 0 . The Wilcox on test was used to test whether SDS- transformed results in the three patient groups significantly deviated from this expected result. Throughout, a $p<0.05$ was considered significant. For these calculations, the SPSS 6.0 software package (SPSS Inc.; Chicago, IL, U.S.A.) was used.

\section{RESULTS}

Anthropometric characteristics of the healthy study population are given in Table 1. Pubertal stages 1 to 5 were found in $64,13,14,9$, and 57 of the girls, and in $74,14,10,14$, and 36 of the boys, respectively.

The relationship between muscle force and measurements of body size is known to follow the general equation: muscle

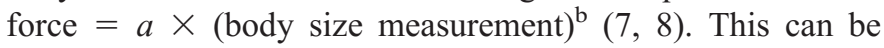


Table 1. Characteristics of healthy study population

\begin{tabular}{ccc}
\hline & Mean \pm SD & Median (range) \\
\hline Girls $(n=157)$ & & \\
Age $(\mathrm{y})$ & $12.0 \pm 3.8$ & $11.5(6.0-19.9)$ \\
Height $(\mathrm{cm})$ & $150.6 \pm 18.0$ & $153.9(110.1-190.6)$ \\
Weight $(\mathrm{kg})$ & $44.5 \pm 17.0$ & $44.5(18.4-100.9)$ \\
MIGF $(\mathrm{N})$ & $185 \pm 83$ & $196(39-392)$ \\
Boys $(n=148)$ & & \\
Age $(\mathrm{y})$ & $11.7 \pm 3.6$ & $11.5(5.9-18.3)$ \\
Height $(\mathrm{cm})$ & $152.8 \pm 21.0$ & $153.7(109.3-193.0)$ \\
Weight $(\mathrm{kg})$ & $46.2 \pm 18.4$ & $45.1(20.6-103.3)$ \\
MIGF $(\mathrm{N})$ & $225 \pm 127$ & $196(49-569)$ \\
\hline
\end{tabular}

conveniently transformed into a linear relationship by logarithmic transformation of both sides of the equation: log (muscle force) $=a+b \times \log$ (body size measurement). For consistency, the logarithmically transformed MIGF was not only used to examine the relationship of MIGF with height and weight, but also with age and pubertal stage.

The pattern of the age-dependent increase in MIGF differed between the sexes (Fig. 1). Age and log MIGF showed a linear association in boys. In girls, the increase in MIGF leveled off after the age of $15 \mathrm{y}$, and the relationship between age and log MIGF was best described by a polynomial of the second order. In contrast, there was a linear relationship between pubertal stage and log MIGF in both sexes. After logarithmic transfor-
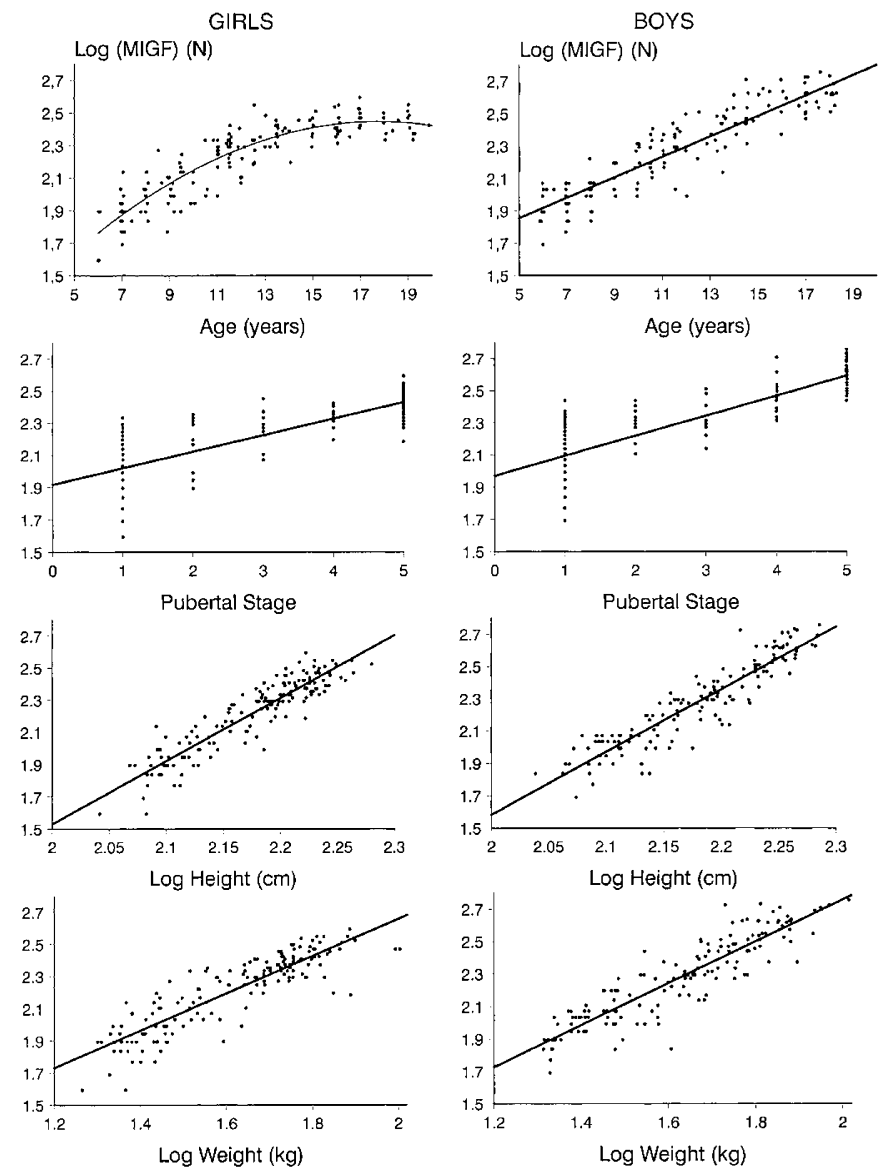

Figure 1. Simple regressions on $\log$ MIGF. The regression lines are indicated. mation, both height and weight were linearly associated with $\log$ MIGF (Fig. 1). The regression equations for these associations are given in Table 2.

Reference data were derived from the regressions between $\log$ MIGF and age and log height. The expected result for log MIGF was derived from these regression equations. The lower limit of the reference range was calculated as the expected result minus $2 \mathrm{SD}$ of the residual. The upper limit corresponds to the expected result plus $2 \mathrm{SD}$. Because the regression was performed on $\log$ MIGF, these values were raised to the power of 10 to obtain the reference range for MIGF. Figure 2 shows these reference ranges together with the original data from which they were derived. There is a close match of the age-dependent reference ranges and the raw data from approximately 7 to $19 \mathrm{y}$ of age in girls and from 7 to $18 \mathrm{y}$ in boys. The height-dependent values appear to be well reflected from 120 to $170 \mathrm{~cm}$ in girls and from 120 to $180 \mathrm{~cm}$ in boys. Tables 3 and 4 give the lower and upper limits of these reference ranges in yearly intervals and in $10-\mathrm{cm}$ steps, respectively.

These reference ranges were used to evaluate MIGF results in kidney transplant recipients, patients with epilepsy who were taking anticonvulsant therapy, and patients suffering from cystic fibrosis (Fig. 3). Six of the 14 transplanted patients (43\%), five of the 13 cystic fibrosis patients (38\%), and none of the patients with epilepsy had an MIGF below the agedependent reference range. MIGF was below the heightdependent reference range in only two of the renal transplant recipients $(21 \%)$, in one patient with cystic fibrosis $(8 \%)$, and in none of the patients with epilepsy.

Descriptive statistics for these results are shown in Table 5 . MIGF was clearly decreased in the kidney transplantation group when related to age, but was normal when related to height ( $p=0.001$ for difference between age- and heightdependent MIGF SDS, Wilcoxon test). This discrepancy can be explained by the short stature of this group of patients. Similarly, MIGF was decreased by age in cystic fibrosis patients, but the height-dependent MIGF SDS was not significantly different from 0 . In contrast to these two groups, height was normal in the patients taking anticonvulsant therapy, and no difference was found between age- and height-related MIGF SDS ( $p=0.94$ for difference between age- and heightdependent MIGF SDS).

Table 2. Simple regression equations predicting log MIGF

\begin{tabular}{lcc}
\hline \multicolumn{1}{c}{ Log MIGF prediction } & SD $^{*}$ & $r^{2}$ \\
\hline Girls & & \\
$0.176 \times$ age $-0.004957 \times \mathrm{age}^{2}+0.886$ & 0.0996 & 0.81 \\
$0.103 \times($ pubertal stage $)+1.917$ & 0.1328 & 0.66 \\
$3.915 \times$ log height -6.300 & 0.0912 & 0.84 \\
$1.157 \times$ log weight +0.344 & 0.1096 & 0.77 \\
Boys & & \\
$0.06356 \times$ age +1.538 & 0.1061 & 0.83 \\
$0.125 \times($ pubertal stage $)+1.970$ & 0.1386 & 0.70 \\
$3.873 \times$ log height -6.163 & 0.0977 & 0.85 \\
$1.289 \times$ log weight +0.180 & 0.1098 & 0.81 \\
\hline
\end{tabular}

* Residual SD of the regression.

Units: MIGF, N; age, y; height, $\mathrm{cm}$; weight, $\mathrm{kg}$; pubertal stage, number between 1 and 5 . 
GIRLS
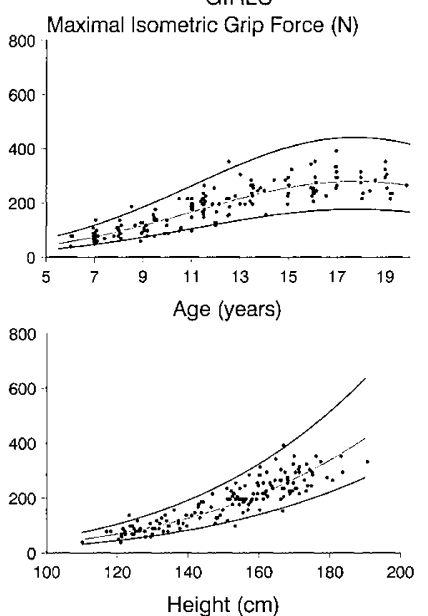

BOYS
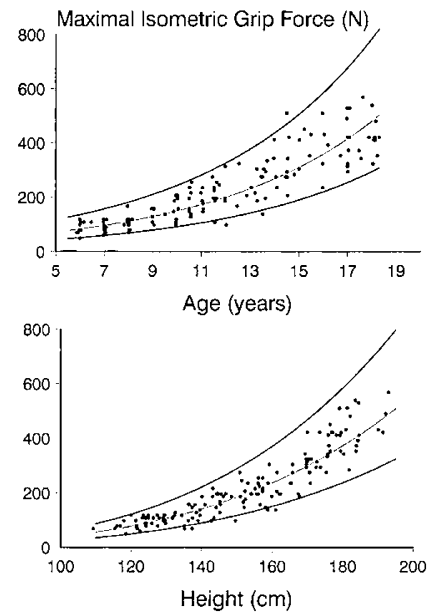

Figure 2. Reference ranges for MIGF and original data. The lower and upper lines represent the lower and upper limits of the reference range, respectively. The middle line indicates the expected value.

Table 3. Reference ranges for MIGF (in N) by age. To convert dynamometer reading (in $\mathrm{kg}$ ) into $\mathrm{N}$, multiply by 9.81

\begin{tabular}{ccc}
\hline Age $(\mathrm{y})$ & Girls & Boys \\
\hline 7 & $47-119$ & $59-157$ \\
8 & $60-150$ & $68-181$ \\
9 & $74-185$ & $79-210$ \\
10 & $89-224$ & $91-243$ \\
11 & $105-264$ & $106-281$ \\
12 & $122-304$ & $123-326$ \\
13 & $137-343$ & $142-377$ \\
14 & $151-378$ & $164-437$ \\
15 & $163-407$ & $190-505$ \\
16 & $171-429$ & $220-585$ \\
17 & $176-441$ & $255-677$ \\
18 & $177-444$ & $295-784$ \\
19 & $174-436$ & - \\
\hline
\end{tabular}

\section{DISCUSSION}

In this study we present pediatric reference data for MIGF using the Jamar dynamometer for clinical use. It is important to note that MIGF reflects only one aspect of muscle function at one anatomic site. Results of the MIGF test therefore cannot be assumed to reflect the functional status of the entire muscle system. The test also does not provide insight into other functionally important measurements of muscle performance, such as contraction velocity and coordination. Yet, in many clinical settings, measuring MIGF will be the only readily available method to obtain at least one quantitative measure of muscle function.

Table 4. Reference ranges for MIGF (in N) by height. To convert dynamometer reading (in $\mathrm{kg}$ ) into $\mathrm{N}$, multiply by 9.81

\begin{tabular}{ccc}
\hline Height $(\mathrm{cm})$ & Girls & Boys \\
\hline 120 & $45-105$ & $49-122$ \\
130 & $62-144$ & $67-166$ \\
140 & $83-193$ & $90-221$ \\
150 & $109-252$ & $117-289$ \\
160 & $140-325$ & $151-370$ \\
170 & $178-412$ & $191-469$ \\
180 & - & $238-585$ \\
\hline
\end{tabular}

GIRLS
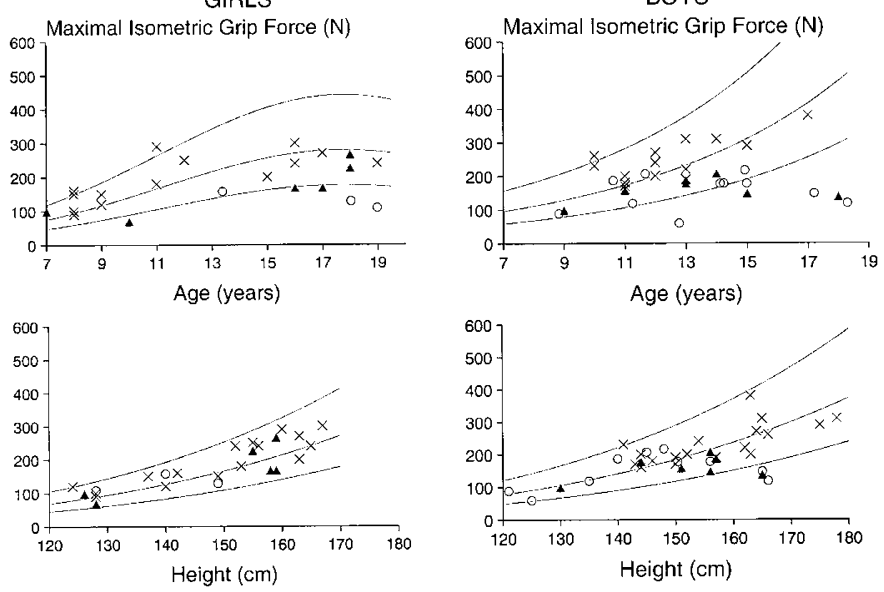

Figure 3. MIGF results in three patient groups. The lower and upper lines represent the lower and upper limits of the reference range, respectively. The middle line indicates the expected value. $(O$, renal transplant recipients; $\Delta$, patients with cystic fibrosis; $\times$, patients with epilepsy who were taking anticonvulsant monotherapy with either valproic acid or carbamazepine.)

The age-dependent results of the present study are similar to those reported for American children and adolescents by Sinaki et al. (6). However, our data are lower than the values reported by other authors from the United States $(3,5)$ or from Australia (4). This may be owing to real variations in MIGF among the populations, or may indicate that children are weaker now than they were $15 \mathrm{y}$ ago, when these studies were performed. Yet, it is also important to note that each of these studies used the dynamometer in a different fashion. Studies vary in the setting of the dynamometer (fixed versus size-adjusted), the position of the arm (supported on a table versus unsupported; angles of elbow and wrist), which side was analyzed (right versus left; dominant versus nondominant), the number of trials (one to three), and how the results were evaluated (mean value of all trials versus best result). Thus, if a given set of reference data is to be used for clinical purposes, it must be ensured that the testing conditions are identical to those described for the reference population.

An important goal of this study was to derive MIGF reference data not only for age but also for height, so that these values could be used to assess children with short stature. It is well known that height — and thereby the lengths of muscles, bones, and lever arms - is the main determinant of upper extremity muscle force (7-9), and this was confirmed by the present results. Although it is also possible to compute reference values as a function of weight, these data are not presented in tabular form, because there is little logic in relating the force of non-weight-bearing muscles to weight.

As exemplified in the three patient groups examined in this study, the present reference material can be used in two different ways. The simplest approach is to compare a patient's test result with the age- and height-dependent reference ranges given in Figure 2 or in Tables 3 and 4. When a more numerical analysis is required, SDS can be calculated using the data given in Table 2. Assume that a 12.0-y-old girl with short stature $(130 \mathrm{~cm})$ has an MIGF of $90 \mathrm{~N}$. According to Table 2, the expected log MIGF for her age is $0.176 \times 12.0-0.004957 \times$ 
Table 5. Results in patient groups

\begin{tabular}{|c|c|c|c|}
\hline Group & Mean \pm SD & Median (range) & $p$ value* \\
\hline \multicolumn{4}{|c|}{ Kidney transplantation group $(n=14)$} \\
\hline Age (y) & $14.2 \pm 3.1$ & $14.2(8.8-19)$ & \\
\hline Height (SDS) & $-2.5 \pm 1.2$ & $-2.2(-5.7--0.9)$ & 0.0001 \\
\hline MIGF (age - SDS) & $-2.5 \pm 1.9$ & $-1.8(-5.9-0.5)$ & 0.0006 \\
\hline MIGF (height - SDS) & $-0.4 \pm 1.5$ & $-0.2(-3.7-1.2)$ & 0.63 \\
\hline \multicolumn{4}{|c|}{ Cystic fibrosis group $(n=13)$} \\
\hline Weight (SDS) & $-1.3 \pm 0.8$ & $-1.5(-2.5-0.1)$ & 0.0005 \\
\hline MIGF (age - SDS) & $-1.6 \pm 1.6$ & $-1.1(-5.1-1.2)$ & 0.006 \\
\hline MIGF (height - SDS) & $-0.5 \pm 1.1$ & $-0.7(-2.9-1.2)$ & 0.17 \\
\hline \multicolumn{4}{|c|}{ Anticonvulsant group $(n=33)$} \\
\hline Age (y) & $12.2 \pm 2.9$ & $12(8-19)$ & \\
\hline Height (SDS) & $0.0 \pm 1.2$ & $-0.3(-2.4-2.8)$ & 0.87 \\
\hline
\end{tabular}

* Indicates significance of SDS from 0.

$12.0^{2}+0.886=2.284$. The actually measured value is $\log 90$ $=1.954$. Her age-dependent MIGF SDS is the difference between measured and expected results divided by the residual SD given in Table 2, corresponding to $(1.954-2.284)$ / $0.0996=-3.31$. Analogous calculations reveal that the height-dependent MIGF SDS in this case is -0.2 . The conclusion from this evaluation is that this girl's muscle weakness can be explained by her statural delay and that her MIGF is adequate for height. Such calculations may appear cumbersome, but the terms given in Table 2 can be easily entered into widely available database software such as Microsoft Excel (Microsoft Corp., Redmond, WA, U.S.A.) to allow for automatic evaluation of MIGF results.

Muscle weakness is a well-recognized problem in adult patients with chronic renal failure $(11,12)$. Muscle force therefore is usually decreased before kidney transplantation (13), but in younger adults tends to increase after successful renal grafting (14). To our knowledge, no study has followed the changes in muscle force in children before and after kidney transplantation. In our study pediatric renal graft recipients were clearly weaker than children of the same age, but this could entirely be attributed to their reduced body height. Thus, there was no indication that the chronic disease process or the therapeutic interventions affected maximal isometric muscle force apart from causing short stature. Obviously, more detailed and longitudinal studies are required to gain more certainty on this topic. The present reference material should facilitate such longitudinal analyses of MIGF in growing individuals.

Muscle weakness is a common finding in patients with cystic fibrosis (15-18). Using the same dynamometer as in the present study, Elkin et al. (18) found that MIGF of young adults with cystic fibrosis was decreased by about 0.9 SD compared with age-matched healthy controls, which is very close to our results. Similar to Elkin et al. (18), we found that this decreased muscle force is largely a size-dependent phenomenon.
We are not aware of any studies that examined muscle force in pediatric patients receiving monotherapy with carbamazepine or valproic acid. This may suggest that there is no clinically apparent problem with the muscle system in these patients, as also appears from our results. In fact, this group of patients was even slightly stronger than their healthy peers.

In conclusion, in this study we present reference data for MIGF in children and adolescents and demonstrate their use in clinical practice. This material should facilitate the analysis of MIGF in clinical and research settings.

Acknowledgment. The authors thank the staff of the Research Institute for Child Nutrition for continuing support.

\section{REFERENCES}

1. Frost HM, Schonau E 2000 The "muscle-bone unit" in children and adolescents: a 2000 overview. J Pediatr Endocrinol Metab 13:571-590

2. Bechtol CO 1954 Grip test: the use of a dynamometer with adjustable handle spacings. J Bone Joint Surg Am 36A:820-824

3. Ager CL, Olivett BL, Johnson CL 1984 Grasp and pinch strength in children 5 to 12 years old. Am J Occup Ther 38:107-113

4. Fullwood D 1986 Australian norms for hand and finger strength of boys and girls aged 5-12 years. Austr Occup Ther J 33:26-36

5. Mathiowetz V, Wiemer DM, Federman SM 1986 Grip and pinch strength: norms for 6 to 19 year-olds. Am J Occup Ther 40:705-711

6. Sinaki M, Limburg PJ, Wollan PC, Rogers JW, Murtaugh PA 1996 Correlation of trunk muscle strength with age in children 5 to 18 years old. Mayo Clin Proc 71:1047-1054

7. Asmussen E, Heeboll-Nielsen K 1955 A dimensional analysis of physical performance and growth in boys. J Appl Physiol 7:593-603

8. Parker DF, Round JM, Sacco P, Jones DA 1990 A cross-sectional survey of upper and lower limb strength in boys and girls during childhood and adolescence. Ann Hum Biol 17:199-211

9. Round JM, Jones DA, Honour JW, Nevill AM 1999 Hormonal factors in the development of differences in strength between boys and girls during adolescence: a longitudinal study. Ann Hum Biol 26:49-62

10. Tanner JM 1962 Growth at Adolescence, 2nd ed. Blackwell, Oxford, pp 25-47

11. Heimburger O, Qureshi AR, Blaner WS, Berglund L, Stenvinkel P 2000 Hand-grip muscle strength, lean body mass, and plasma proteins as markers of nutritional status in patients with chronic renal failure close to start of dialysis therapy. Am J Kidney Dis 36:1213-1225

12. Fahal IH, Bell GM, Bone JM, Edwards RH 1997 Physiological abnormalities of skeletal muscle in dialysis patients. Nephrol Dial Transplant 12:119-127 
13. Bohannon RW, Hull D, Palmeri D 1994 Muscle strength impairments and gait performance deficits in kidney transplantation candidates. Am J Kidney Dis 24:480485

14. Nyberg G, Hallste G, Norden G, Hadimeri H, Wramner L 1995 Physical performance does not improve in elderly patients following successful kidney transplantation. Nephrol Dial Transplant 10:86-90

15. Mier A, Redington A, Brophy C, Hodson M, Green M 1990 Respiratory muscle function in cystic fibrosis. Thorax 45:750-752
16. Lands LC, Heigenhauser GJ, Jones NL 1993 Respiratory and peripheral muscle function in cystic fibrosis. Am Rev Respir Dis 147:865-869

17. Boas SR, Joswiak ML, Nixon PA, Fulton JA, Orenstein DM 1996 Factors limiting anaerobic performance in adolescent males with cystic fibrosis. Med Sci Sports Exerc 28:291-298

18. Elkin SL, Williams L, Moore M, Hodson ME, Rutherford OM 2000 Relationship of skeletal muscle mass, muscle strength and bone mineral density in adults with cystic fibrosis. Clin Sci (Colch) 99:309-314 